\title{
BOUNDARY BEHAVIOUR OF HARMONIC FUNCTIONS AND SOLUTIONS OF PARABOLIC SYSTEMS
}

\author{
by N. A. WATSON
}

(Received 28th October 1986)

\section{Boundary behaviour of harmonic functions}

In [1], Calderón proved that, if $u$ is a harmonic function on $\left.\mathbf{R}^{n} \times\right] 0, \infty[$, and at each point $\xi$ of a subset $E$ of $\mathbf{R}^{n}, u$ is bounded in some cone with vertex $(\xi, 0)$, then $u$ has a nontangential limit at almost every point of $E \times\{0\}$. The main result of this note is a stronger version of this theorem, in which the hypotheses remain unchanged but the nontangential limits in the conclusion are replaced by limits through the more general approach regions first considered by Nagel and Stein in [7].

If $\xi \in \mathbf{R}^{\boldsymbol{n}}, \gamma>0$ and $0<b \leqq \infty$, we denote by $\Gamma_{\gamma}^{b}(\xi)$ the truncated (if $\left.b<\infty\right)$ open cone in $\left.\mathbf{R}^{n} \times\right] 0, b\left[\right.$ with vertex $(\xi, 0)$, aperture $\gamma$, and axis orthogonal to $\mathbf{R}^{n} \times\{0\}$, that is,

$$
\Gamma_{\gamma}^{b}(\xi)=\{(x, t):\|x-\xi\|<\gamma t, 0<t<b\} .
$$

Here, and subsequently, $\|y\|$ denotes the euclidean norm of a point $y \in \mathbf{R}^{n}$.

Throughout this note, we use $m$ to denote Lebesgue measure on $\mathbf{R}^{n}$, and $\Omega$ to denote an open subset of $\left.\mathbf{R}^{n} \times\right] 0, \infty$ [ with the following properties.

(i) There exists $\alpha>0$ such that, whenever $(x, t) \in \Omega$,

$$
\{(y, s):\|y-x\|<\alpha(s-t)\} \subseteq \Omega .
$$

(ii) There exists $\beta>0$ such that, for all $t>0$,

$$
m\left(\left\{x \in \mathbf{R}^{n}:(x, t) \in \Omega\right\}\right) \leqq \beta t^{n} .
$$

(iii) The point $(0,0)$ is a limit point of $\Omega$.

The simplest example of such a set $\Omega$ is the cone $\Gamma_{\alpha}^{\infty}(0)$. Nagel and Stein [7, p. 98] give examples in which $\Omega$ is not contained in any cone, and indeed show that $\Omega$ may contain a sequence which approached $(0,0)$ with any prescribed degree of tangency to $\mathbf{R}^{n} \times\{0\}$.

For each $\xi \in \mathbf{R}^{n}$ we put $\Omega_{\xi}=\Omega+\{(\xi, 0)\}$. We say that a function $u$ on $\left.\mathbf{R}^{n} \times\right] 0, \infty[$ has an $\Omega$-limit $l$ at a point $\xi \in \mathbf{R}^{n}$ if to each $\varepsilon>0$ there corresponds $\delta>0$ such that

$$
|u(x, t)-l|<\varepsilon \text { whenever } \quad(x, t) \in \Omega_{\xi} \quad \text { and } \quad\|x-\xi\|+t<\delta .
$$

Nagel and Stein proved results which, as they pointed out in [7, p. 99], imply that the 
Poisson integral of any function $f$ in $L^{p}\left(\mathbf{R}^{n}\right)$, where $1 \leqq p \leqq \infty$, has an $\Omega$-limit $f(\xi)$ at almost every $\xi \in \mathbf{R}^{n}$. If

$$
P(z, t)=\left(2 / \sigma_{n+1}\right) t\left(\|z\|^{2}+t^{2}\right)^{-(n+1) / 2}
$$

for all $\left.(z, t) \in \mathbf{R}^{n} \times\right] 0, \infty\left[\right.$, where $\sigma_{n+1}$ denotes the surface area of the unit sphere in $\mathbf{R}^{n+1}$, then the Poisson integral $u$ of a signed measure $\mu$ is defined by the convolution

$$
u(x, t)=\int_{\mathbf{R}^{n}} P(x-y, t) d \mu(y)
$$

for all $\left.(x, t) \in \mathbf{R}^{n} \times\right] 0, \infty[$, provided that

$$
\int_{\mathbf{R}^{n}}\left(1+\|y\|^{2}\right)^{-(n+1) / 2} d|\mu|(y)<\infty
$$

(see [4, Theorem 6]. The Poisson integral of a function $f$ is that of the measure $\mu$ given by $d \mu(y)=f(y) d y$. Whenever (2) holds, (1) represents a harmonic function.

An easy consequence of the Nagel-Stein theorem is that, if $u$ is the Poisson integral on $\left.R^{n} \times\right] 0, \infty\left[\right.$ of a function $f$, then $u$ has an $\Omega$-limit $f(\xi)$ at almost every $\xi \in \mathbf{R}^{n}$. This is proved by applying that theorem to the restriction of $f$ to an arbitrary ball in $\mathbf{R}^{n}$.

Our main theorem can now be proved. In fact, this can be done by making only minor alterations to the proof of Calderón's result given by Stein in [8, Chapter VII]. It is the form of the theorem which is interesting, not its proof, since the boundedness of $u$ is required only on cones, not on the sets $\Omega_{\xi}$ or any simple variant of them.

Theorem 1. Let $u$ be harmonic on $\left.\mathbf{R}^{n} \times\right] 0, \infty\left[\right.$, and let $E$ be the set of all $\xi \in \mathbf{R}^{n}$ with the property that there exist positive numbers $\gamma, M$ and $b$, such that

$$
|u(x, t)| \leqq M \quad \text { for all } \quad(x, t) \in \Gamma_{y}^{b}(\xi) .
$$

Then $u$ has $\Omega$-limits a.e. on $E$.

Proof. Follow Stein's proof, but note that the Poisson integrals therein have $\Omega$-limits a.e.

Theorem 1 can be used to extend the Nagel-Stein theorem to arbitrary Poisson integrals.

Theorem 2. If $u$ is the Poisson integral on $\left.\mathbf{R}^{n} \times\right] 0, \infty[$ of a signed measure $\mu$, and $f$ is the Radon-Nikodym derivative of $\mu$, then $u$ has an $\Omega$-limit $f(\xi)$ at almost every $\xi \in \mathbf{R}^{n}$.

Proof. It is well-known that $u$ has a nontangential limit $f(\xi)$ at almost every $\xi \in \mathbf{R}^{n}$ [3, Theorem 4.1]. Hence, given any positive numbers $b$ and $\gamma, u$ is bounded on $\Gamma_{\gamma}^{b}(\xi)$ for almost all $\xi \in \mathbf{R}^{n}$. Therefore $u$ has $\Omega$-limits a.e. on $\mathbf{R}^{n}$, by Theorem 1 . That these limits are equal to $f$ a.e. follows because $\Gamma_{a}^{\infty}(0) \subseteq \Omega$.

Theorem 2 has been proved by a different method in [6]. 


\section{Analogues for parabolic systems}

The Nagel-Stein theorem has an analogue for solutions of homogeneous parabolic systems in the sense of Petrowskii, of order 2 , defined on a strip $\mathbf{R}^{n} \times[0, T]$. Such systems are described in [2, Section 1], and the conditions on the coefficients given therein are sufficient to enable us to prove this analogue. The Poisson integral of a function $f \in L^{p}\left(\mathbf{R}^{n}\right)$ is replaced by the formula [2,(15)], with each $f_{j} \in L^{p}\left(\mathbf{R}^{n}\right)$. In the conditions on $\Omega, \alpha(s-t)$ is replaced by $\alpha(\sqrt{ } s-\sqrt{ } t)$ in (i), and $\beta t^{n}$ by $\beta t^{n / 2}$ in (ii). The first step in the proof is to use the upper estimate for the elements of the fundamental solution given in [5, Chapter 9], but then the proof proceeds analogously to the harmonic case. The crucial estimate of the maximal function follows from [7, Theorem 2] by square rooting the exceptional variable. These results were obtained in collaboration with Dr. J. Chabrowski.

Analogues of Theorems 1 and 2 can also be obtained, but their proofs require that the elements of the fundamental solution are non-negative, so that the parabolic system must be weakly coupled [2, Section 4]. A lower estimate for the diagonal elements of the fundamental solution is also required. The analogue of Theorem 1 can be proved, under the same conditions on the coefficients as were imposed in [9], by modifying the proof of the theorem therein in the same way that the proof for the harmonic case was modified above. The cones must, of course, be replaced by paraboloids. For Theorem 2, the Poisson integral of a signed measure is replaced by the formula [2, (31)], with signed measures $\mu_{j}$ which satisfy

$$
\int_{\mathbb{R}^{n}} \exp \left(-\kappa\|x\|^{2}\right) d\left|\mu_{j}\right|(x)<\infty
$$

for some constant $\kappa$. The proof carries over easily, with the nontangential limits replaced by the parabolic limits of [2, Section 3]. The conditions on the coefficients are the same as those for the analogue of Theorem 1. An apparently different, but in fact equivalent, analogue of Theorem 2 for a single parabolic equation, is given in [6].

\section{REFERENCES}

1. A. P. Calderon, On the behaviour of harmonic functions near the boundary, Trans. Amer. Math. Soc. 68 (1950), 47-54.

2. J. Chabrowski, Representation theorems for parabolic systems, J. Austral. Math. Soc. (Ser. A) 32 (1982), 246-288.

3. J. L. Dоoв, Relative limit theorems in analysis, J. Anal. Math. 8 (1960/61), 289-306.

4. T. M. FLETT, On the rate of growth of mean values of holomorphic and harmonic functions, Proc. London Math. Soc. (3) 20 (1970), 749-768.

5. A. Friedman, Partial differential equations of parabolic type (Prentice-Hall, Englewood Cliffs, 1964).

6. B. A. Mair and D. Singman, A generalized Fatou theorem, Trans. Amer. Math. Soc. 300 (1987), 705-720. 
7. A. NAgel and E. M. Stein, On certain maximal functions and approach regions, Advances Math. 54 (1984), 83-106.

8. E. M. Stein, Singular integrals and differentiability properties of functions (Princeton University Press, Princeton, 1970).

9. N. A. WAtson, Parabolic limits of solutions of weakly coupled parabolic systems, J. Math. Anal. Appl. 95 (1983), 278-283.

Department of Mathematics

University of Canterbury

Christchurch

New Zealand 